[0212-7199 (2005) 22: 1; pp 4-8] ANALES DE MEDICINA INTERNA Copyright (C) 2005 ARAN EDICIONES, S.L.

AN. MED. INTERNA (Madrid) Vol. 22, N. ${ }^{\circ} 1$, pp. 4-8, 2005

\title{
Morbilidad hospitalaria aguda de las personas mayores de Andalucía
}

\author{
C. GARCÍA ORTEGA ${ }^{1,2}$, J. ALMENARA BARRIOS ${ }^{1,2}$, J. L. GONZÁLEZ \\ CABALLERO $^{3}$, J. L. PERALTA SÁENZ
}

Servicio de Admisión y Documentación. Hospital del Servicio Andaluz de Salud. Algeciras. Cádiz. ${ }^{1}$ Área de Medicina Preventiva y Salud Pública. ${ }^{2}$ Departamento de Estadística. Universidad de Cádiz.

\author{
HOSPITAL MORBILITY OF EDERLY POPULATION IN ANDALUCÍA \\ $(S P A I N)$
}

\section{RESUMEN}

Introducción: En los últimos años asistimos en España a un incremento importante del número de las personas mayores, siendo el objetivo del presente trabajo estudiar la morbilidad hospitalaria de los ancianos.

Material y método: Hemos estudiado la morbilidad en las personas mayores de 65 años ingresadas en los hospitales del sistema sanitario público andaluz perteneciente al Sistema Nacional de Salud de España, analizando el conjunto mínimo básico de datos al alta hospitalaria (CMBDH). En un periodo de tiempo que abarca desde enero de 1998 a diciembre 1999, ambos inclusive.

Resultados: En los 32 hospitales estudiados se registraron un total de 25.255 altas hospitalarias de personas mayores de 65 años. Que se se traduce en una índice anual de hospitalización para este grupo de edad del $15,2 \%$ (IC95\% 15,1-15,3).

Los motivos de ingreso más frecuentes son: las enfermedades del aparato circulatorio $(20,7 \%)$, enfermedades del aparato respiratorio $(14,0 \%)$, enfermedades del sistema digestivo $(11,6 \%)$

Conclusiones: Los ancianos de Andalucía (España) poseen unos índices elevados de morbilidad hospitalaria, especialmente a partir de los 75 años, con importantes variaciones entre las distintas provincias que componen la región andaluza . Los ingresos de estas personas están provocados fundamentalmente por las enfermedades crónicas de los países desarrollados.

PALABRAS CLAVE: Ancianos. Morbilidad hospitalaria. Mortalidad hospitalaria. Conjunto mínimo básico de datos. Grupos de diagnósticos relacionados. Case mix.

\begin{abstract}
Introduction: In recent years we have seen in Spain a significant increase in the number of old people. The aim of this work was to determine hospital morbidity among the elderly.

Materials and methods: We studied the morbidity among patients older than 65 years admitted at the hospitals of our Andalusian public health system that belongs to the Spanish National Health System. We analysed the minimal basic data set at discharge (MBDSD) over a period from January 1998 to December 1999, both inclusive.

Results: In the 32 hospitals studied, a total of 25,255 hospital discharges of patients older than 65 years were recorded. This means an annual hospitalization rate in this age group of $15.2 \%$ (95 \% CI: 15.115.3).

The most frequent causes of hospitalisation are: diseases of the circulatory system (20.7\%), diseases of the respiratory system (14.0\%), diseases of the digestive system (11.6\%).

Conclusions: The old population of Andalusia (Spain) has a high rate of hospital morbidity, specially for people older than 75 years, and significant variations are seen between the different provinces of the Andalusian Region. Their hospitalization is due mainly to chronic diseases of developed countries.
\end{abstract}

KEY WORDS: Elderly. Hospital morbidity. Hospital mortality. Minimal basic data set. Diagnosis-related groups. Case mix.

García Ortega C, Almenara Barrios J, González Caballero JL, Peralta Sáenz. JL. Morbilidad hospitalaria aguda de las personas mayores de Andalucía. An Med Interna (Madrid) 2005; 22: 4-8.

\section{INTRODUCCIÓN}

Los cambios demográficos producidos en España en los últimos años son similares al resto de los países desarrollados de nuestro entorno: incremento de la proporción de personas mayores y un descenso del porcentaje de jóvenes. El censo de 2001 (1), estimaba la población española mayor de 65 años en 6.964.267 individuos, que representan el $17 \%$ del total de los españoles. Las proyecciones demográficas indican que estos cambios se van a mantener e incluso se acentuarán en el futuro, produciéndose un preocupante envejecimiento de la población (2), con consecuencias importantes en numerosas áreas, siendo una de las más afectadas el sistema sanitario y el sistema de protección social $(3,4)$. Para comprender la problemática de este grupo de edad se ha de tener en cuenta; que más del $20 \%$ de esta población vive sola, que su nivel cultural y edu-

Trabajo aceptado: 30 de septiembre de 2004

Correspondencia: Cesáreo García Ortega. Servicio de Atención al Usuario. Hospital del SAS. Ctra. de Getares, s/n. 11207 Algeciras (Cádiz) www.cesareo.garcia.sspa@juntadeandalucia.es 
cativo es muy inferior al de las generaciones siguientes, y que casi un $10 \%$ presenta algún grado de deterioro cognitivo (5).

Existen dos teorías contradictorias sobre el impacto que sobre la salud traerá el incremento en la duración de la vida y la disminución de la mortalidad en edades avanzadas (2). La primera de ellas, denominada expansión de la morbilidad establece que la consecuencia de la reducción de las tasas de mortalidad será el prolongarla prolongación del tiempo durante el cual las enfermedades crónicas y las incapacidades se manifestarán, provocando un aumento en su prevalencia.

La segunda de ellas, conocida como teoría de compresióncomprensión de la mortalidad, indica que la adopción de estilos de vida saludables y los avances en la medicina, provocarán no solo una reducción en las tasas de mortalidad, sino del tiempo en que estas se manifiestan. La evidencia científica actual, con el análisis de la evolución de los resultados de la encuesta National Long-term Care Survey, de Estados Unidos, permite afirmar que la teoría de compresión de la morbilidad es la más plausible $(6,7)$.

Las personas de edad avanzada plantean numerosos problemas específicos a los servicios sanitarios, a los que se le suman los derivados de un escaso desarrollo de la red de atención social. El sistema sanitario y muy específicamente el hospital, ha pasado de atender a pacientes jóvenes con patología aguda a pacientes con patologías crónicas no resolubles definitivamente en el estado actual de la sanidad. Esto exige cambiar la oferta de un sistema que ofrece soluciones a episodios (patología aguda de aparición inesperada) a una gestión continua de los problemas sociosanitarios (pluripatología crónica de evolución previsible) (8).

Sin olvidar en ningún momento la necesidad del abordaje holístico de la persona anciana, el presente trabajo estará centrado en los aspectos sanitarios. Para poder estimar la necesidad de servicios y/o cuidados sanitarios en las personas mayores es imprescindible conocer el estado de salud de los mismos y especialmente la prevalencia de aquellas patologías que se asocian a mayor riesgo de hospitalización. Por todo ello, el objetivo del presente trabajo es estudiar la morbilidad hospitalaria de los ancianos, identificando las enfermedades que determinan los ingresos hospitalarios, como punto de partida de programas preventivos y/o de tratamiento que eviten o disminuyan los mismos o sus consecuencias asociadas.

\section{MATERIAL Y MÉTODO}

La fuente de información fue el conjunto mínimo básico de datos al alta hospitalaria (CMBDH), que puede definirse como un conjunto de variables obtenidas en el momento del alta, que proporcionan información sobre el paciente, su entorno, la institución que lo atiende y su proceso asistencial. El CMBDH extracta la información del episodio de hospitalización, recogiendo datos administrativos, demográficos y clínicos (9).

Se realizó un estudio de la morbi-mortalidad hospitalaria usando la información disponible al alta de los 32 hospitales públicos (17.210 camas) de Andalucía (7.305.117 habitantes), mediante el CMBDH. Se excluyeron los hospitales de enfermos crónicos.

El periodo de estudio incluye las altas producidas entre enero de 1998 a diciembre de 1999. Se estudiaron las características personales (edad, sexo y lugar de residencia) de los pacientes ingresados y las características del episodio de hospitalización (forma de ingreso, diagnóstico, procedimiento, servicio, causa y destino del alta).

El análisis estadístico se baso en la descripción univariante, mediante las distribuciones de frecuencias oportunas. La evaluación de hipótesis se llevó a cabo mediante los contrastes oportunos paramétricos o no paramétricos. El nivel de confianza se estableció en un 95\%. El programa de análisis usado fue SPSS versión 10.0.

\section{RESULTADOS}

En 1998 y 1999 se registraron en Andalucía 308.052 altas hospitalarias en personas mayores de 65 años. Estos ingresos se traducen en una índice anual de hospitalización para las personas mayores del 15,2 \% (IC95\% 15,1-15,3). La frecuentación del género masculino 18,6\% (IC95\% 18,5-18,7) fue significativamente mayor que la del femenino $12,7 \%$ (IC $95 \%$ 12,6-12,8). La edad media de los pacientes fue 74,2 años (DE 6,4 años) en los hombres, y de 75,9 años (DE 0,2 años) en las mujeres $(\mathrm{p}<0,01)$.

Existen diferencias importantes al examinar la distribución por provincias de la frecuentación hospitalaria. Con valores que pueden oscilan entre un índice anual de hospitalización del 14,4 \% en Málaga a un índice del 32,7 \% en Almería. Fenómeno que puede ser explicado por la intensa correlación estadística observada entre el número de camas y el índice de frecuentación. ( $\mathrm{r}$ de Pearson $=0,91, \mathrm{p}=0,002$ ).

La distribución por géneros en los diferentes servicios no es uniforme. Exceptuando como es lógico ginecología, los servicios con mayor proporción de ingresos femeninos son traumatología, reumatología y endocrinología. Los servicios con mayor proporción de ingresos masculinos son: urología, ORL y neumología.

Los motivos de ingreso más frecuentes son las enfermedades del aparato circulatorio $(20,7 \%)$, enfermedades del sistema digestivo $(18,4 \%)$ y enfermedades del aparato respiratorio $(11,8 \%)$. Este orden se mantiene inalterable al considerar el número de días de hospitalización causados. Se observan diferentes patrones de enfermar por sexos: los hombres presentan un mayor porcentaje de ingresos en las enfermedades de los siguientes aparatos: respiratorio, urinario, digestivo y circulatorio. En las mujeres el mayor porcentaje lo ostentan los siguientes aparatos: musculoesquelético, hepatobiliar, reproductor y mama (Fig. 1).

La estancia media de los pacientes fue de 13,3 días (D.E. 13,2 días), oscilando entre los 3,4 días de oftalmología hasta los 16,5 días de cirugía cardiovascular. La comorbilidad en los ingresos de las personas mayores es lógicamente frecuente. Y así, el número medio de diagnósticos notificados por alta fue de 3,4 (DE 1,2).

Los diagnósticos principales de ingreso están constituidos por la insuficiencia cardiaca (13.344 ingresos), reagudizaciones de bronquitis crónica (11.283), fracturas de cuello de fémur (10.708), intervenciones de cataratas (9.898) e infarto agudo de miocardio (8.785) (Tabla I). Se evidencia como un número reducido de grupos de patologías agrupan la mayor parte de los ingresos hospitalarios.

Se han producido un total de 14.572 altas por fallecimiento (Tabla II), que se traducen en un índice de mortalidad intrahospitalaria del 8,6 \%. El número de hombres fallecidos 

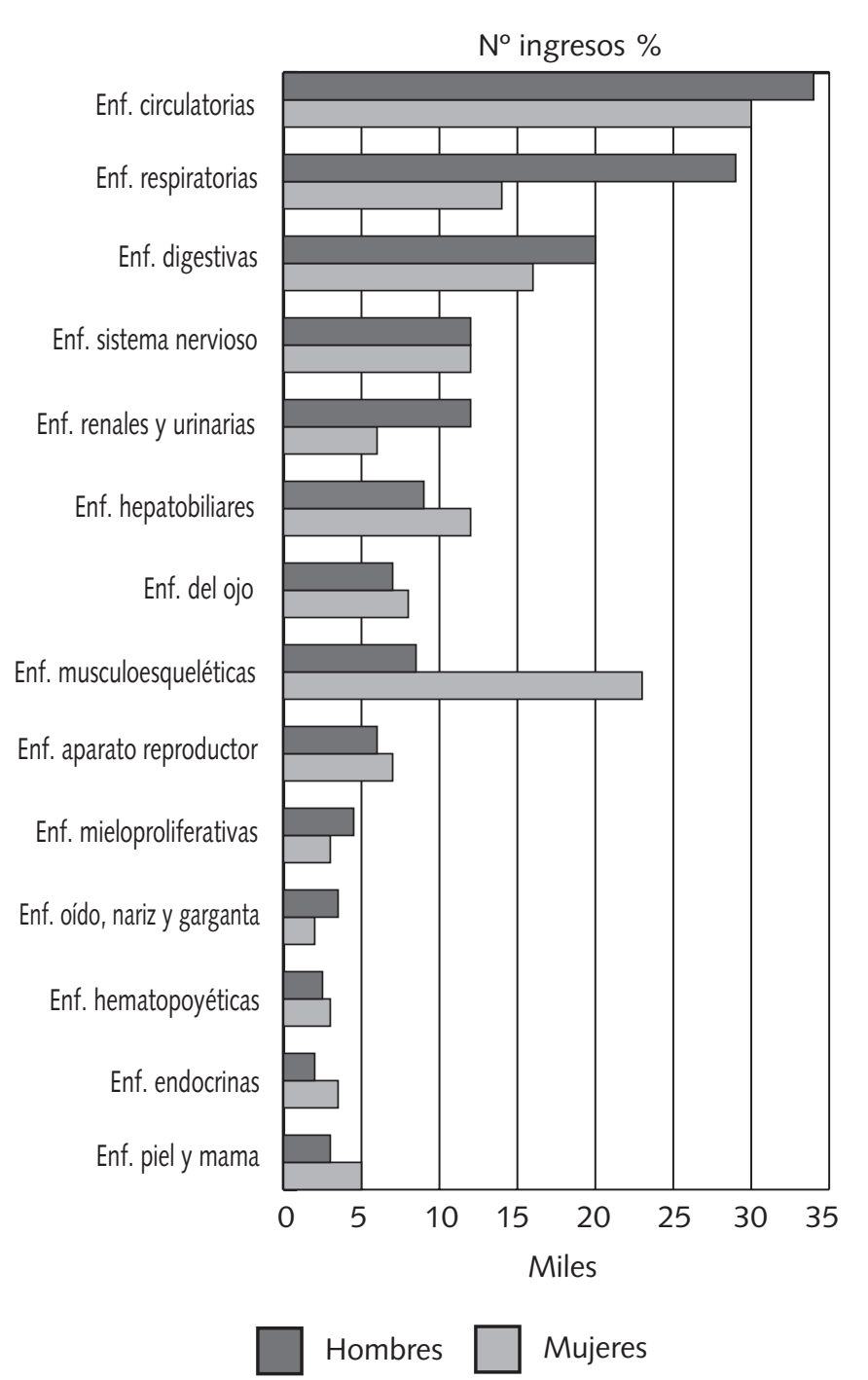

Fig. 1. Ingresos según el género en las principales categorías diagnósticas mayores (porcentaje respecto al total de ingresos por género).

( $n=14.572$, mortalidad hospitalaria del 9,1\%) es significativamente mayor que los exitus en las mujeres $(n=11.943$, mortalidad hospitalaria del 8,0\%). En los hombres fueron las exacerbaciones de la bronquitis crónica la primera causa de mortalidad, seguidas del infarto de miocardio y la insuficiencia cardiaca. En las mujeres la principal causa de mortalidad hospitalaria fue la insuficiencia cardiaca, seguida del infarto de miuocardio y de la trombosis cerebral.

\section{DISCUSIÓN}

Si bien en las personas mayores habitualmente es difícil encontrar una sola causa responsable del ingreso, las enfermedades identificadas con mayor frecuencia como diagnóstico principal de ingreso han sido las relacionados con el aparato circulatorio, respiratorio, digestivo y las neoplasias, en con-
TABLA I

DIAGNÓSTICO PRINCIPAL DE INGRESO MÁS FRECUENTE EN LAS PERSONAS MAYORES DE 65 AÑOS, NÚMERO DE CASOS Y PORCENTAJE DE INGRESOS RESPECTO A ESTE GRUPO DE EDAD

\begin{tabular}{|c|c|c|c|c|}
\hline CIE-9-MC & Diagnóstico & $N^{0}$ & $\%$ & $\begin{array}{c}\% \\
\text { acumulado }\end{array}$ \\
\hline 366 & Insuficiencia cardíaca & 13.344 & 4,3 & 4,3 \\
\hline 491 & Bronquitis crónica & 11.283 & 3,7 & 8 \\
\hline 820 & Fractura de cuello de fémur & 10.708 & 3,5 & 11,5 \\
\hline 366 & Cataratas & 9.898 & 3,2 & 14,7 \\
\hline 410 & Infarto agudo de miocardio & 8.785 & 2,9 & 17,5 \\
\hline 574 & Colelitiasis & 8.728 & 2,8 & 20,4 \\
\hline 434 & Oclusión arterias cerebrales & 7.102 & 2,3 & 22,7 \\
\hline 411 & Angor inestable & 6.722 & 2,2 & 24,9 \\
\hline 486 & $\begin{array}{l}\text { Neumonía, organismo no } \\
\text { especificado }\end{array}$ & 6.407 & 2,1 & 26,9 \\
\hline 715 & Osteoartrosis & 5.025 & 1,6 & 28,6 \\
\hline 188 & Neoplasia maligna de vejiga & 4.982 & 1,6 & 30,2 \\
\hline 250 & Diabetes & 4.733 & 1,5 & 31,7 \\
\hline 427 & Disritmias cardiacas & 4.705 & 1,5 & 33,3 \\
\hline 550 & Hernia inguinal & 4.484 & 1,5 & 34,7 \\
\hline V58 & Ingreso para quimioterapia & 4.423 & 1,4 & 36,2 \\
\hline 996 & $\begin{array}{l}\text { Complicaciones procedimientos } \\
\text { quirúrgicos }\end{array}$ & 4.267 & 1,4 & 37,5 \\
\hline 413 & Angina de pecho & 3.728 & 1,2 & 38,7 \\
\hline 414 & Cardiopatía isquémica crónica & 3.718 & 1,2 & 40,0 \\
\hline 577 & Enfermedades del páncreas & 3.664 & 1,2 & 41,1 \\
\hline 600 & Hipertrofia de próstata & 3.528 & 1,1 & 42,3 \\
\hline
\end{tabular}

cordancia con otros estudios (10-13). Todas ellas enfermedades crónicas de los países desarrollados, en cuya etiología hay asociados factores susceptibles de prevención como: el habito de fumar la hipercolesterolemia, la hipertensión y la inactividad física.

La cardiopatía isquémica, con incidencia creciente conforme avanza la edad y con predominio en el sexo masculino, clínicamente puede presentarse en el paciente de edad avanzada como ángor en cualquiera de sus formas, infarto, insuficiencia cardiaca o muerte súbita. Si bien en la actualidad asistimos a un interés creciente en la obtención de datos disponibles sobre la incidencia real de la cardiopatía isquémica en nuestro país llama la atención la escasez de datos sobre la incidencia, mortalidad y letalidad del infarto de miocardio en los ancianos Así mismo, últimamente se ha evidenciado la necesidad de realizar estudios sobre la efectividad de los tratamientos cardiovasculares en las personas mayores, ya que se evidencia una escasa representación de las mismas en los ensayos clínicos que evalúan este tipo de fármacos (14).

La insuficiencia cardiaca y la enfermedad cardiaca hipertensiva constituyen una importante causa de morbimortalidad hospitalaria en las personas de edad avanzada. Con los criterios diagnósticos actuales en los pacientes geriátricos la hipertensión alcanza una prevalencia cercana al 70\% (15). En los últimos años asistimos a un incremento en los ingresos por insuficiencia cardiaca en nuestro país, situación relacionada 
TABLA II

MORTALIDAD HOSPITALARIA POR GÉNERO EN LOS PACIENTES MAYORES DE 65 AÑOS

\begin{tabular}{lrlr}
\hline \multicolumn{1}{c}{ Hombres } & \multicolumn{2}{c}{ Mujeres } \\
\hline Diagnóstico principal & Casos & Diagnóstico principal & Casos \\
\hline Bronquitis crónica & 885 & Insuficiencia cardiaca & 1.042 \\
Infarto agudo de miocardio & 777 & Infarto agudo de miocardio & 648 \\
Insuficiencia cardiaca & 722 & Oclusión de las arterias cerebrales & 532 \\
Neo. maligna de bronquios y pulmón & 664 & Accidente cerebro-vascular & 485 \\
Neumonía, organismo no especificado & 599 & Neumonía, organismo no especificado & 416 \\
Oclusión arteria cerebral & 477 & Fractura cuello fémur & 372 \\
Hemorragia intracerebral & 335 & Otras enfermedades pulmonares & 305 \\
Otras enfermedades pulmonares & 322 & Hemorragia intracerebral & 271 \\
Enf. cerebro vascular aguda mal definida & 316 & Diabetes mellitus & 268 \\
Neo. secundaria respiratoria o digestiva & 222 & Exacerbaciones de bronquitis crónica & 201 \\
Resto & 9.253 & Resto & 7.403 \\
Total & 14.572 & Total & 11.943 \\
\hline
\end{tabular}

Neo: neoplasia

con el envejecimiento y con el aumento de la supervivencia tras el infarto de miocardio (16).

La hipertensión es el principal factor de riesgo de la enfermedad cerebrovascular, que se manifiesta con elevadas tasas de ingreso y mortalidad en estas edades; patología con importante repercusión social por las consecuencias asistenciales y económicas que de ella derivan $(17,18)$.

La patología respiratoria se encuentra representada en estas edades por la patología infecciosa (exacerbaciones de bronquitis crónicas, EPOC y neumonías) y la patología tumoral. Sabemos que la mayor parte de la patología respiratoria se encuentra asociada al uso del tabaco, lo que explicaría el claro predominio del sexo masculino en los servicios de neumología estudiados.

La neumonía adquirida en la comunidad es una enfermedad frecuente, que presenta una alta comorbilidad, condicionando un número considerable de estancias hospitalarias, con unos costes elevados y alta tasa de mortalidad $(19,20)$. A la luz de los conocimientos actuales se espera que la inmunización de las personas de edad avanzada contra los virus de la influenza y neumococos pueda disminuir la incidencia y la gravedad de las infecciones de las vías respiratorias (21).

La patología quirúrgica de los ancianos está reunida en un número de diagnósticos reducido: cataratas, patología biliar, fracturas de cuello de fémur, hernia inguinal, complicaciones de procedimientos quirúrgicos previos, la hipertrofia de próstata, las neoplasias de colon y patología del útero y sus estructuras de soporte. Dentro de este grupo, un porcentaje importante de la cirugía del ojo y de las hernias de la pared abdominal se realizan en régimen ambulatorio (por lo que no están contabilizadas como ingresos hospitalarios), que se muestra como una alternativa segura en el tratamiento de muchas patologías quirúrgicas del paciente anciano (22).

Las caídas constituyen la principal causa de accidentes mortales y no mortales entre los ancianos en los países desarrollados (23). La proporción de caídas que provocan fracturas es baja, pero el número absoluto de ancianos que sufren fracturas es elevado, ocasionando una gran demanda asistencial. Las fracturas de cadera, con incidencia creciente (por lo que se prevé un incremento importante de las mismas en un futuro) (24), son mas frecuentes en las mujeres y ocasionan una elevada morbimortalidad. Su etiología es multifactorial, aunque dos son los factores principales: las caídas y la debilidad ósea. En su prevención, junto a la prevención de los accidentes (25), el arma eficaz es evitar la aparición de osteoporosis (dieta, ejercicio físico, control de los factores de riesgo) o el tratamiento de la misma una vez que esta aparece (26).

Dentro de las limitaciones de este trabajo hemos de destacar, para conocer la verdadera morbilidad en estas personas, que no toda la actividad especializada se produce dentro del hospital, encontrándonos en la actualidad frente a un importante desarrollo de formas alternativas a la hospitalización clásica, tales como el hospital de día médico o la cirugía mayor ambulatoria. Así mismo, hemos de recordar que numerosos problemas de salud de los ancianos serán atendidos por la medicina de familia y nunca acudirán al hospital.

Así mismo, no se ha ejercido ninguna restricción sobre los reingresos (estimados entre el 2,5-4\%) (12), que puede condicionar una sobrestimación de la prevalencia de determinadas patologías. Se ha preferido este planteamiento al estar cuantificando utilización de los servicios hospitalarios, es decir episodios de hospitalización.

En resumen, los motivos de ingreso hospitalario están constituidos fundamentalmente por las enfermedades crónicas de los países desarrollados, que ya se manifestaban habitualmente en la edad adulta y donde la medicina preventiva se ha mostrado como el arma más eficaz para combatirlas. En este sentido, la Organización Panamericana de la Salud (OPS) indica "que el buen envejecimiento depende en gran medida de la prevención de las enfermedades y la discapacidad, el mantenimiento de una gran actividad física y de las funciones cognoscitivas y la participación ininterrumpida en actividades sociales y productivas" (27). 


\section{Bibliografía}

1. Instituto Nacional de Estadística. Censo de población 2001 Ministerio del Interior. Madrid, 2002

2. Casado D, López i Casasnovas G. Vejez, dependencia y cuidados de larga duración. Situación actual y perspectivas de futuro. Barcelona, Fundación la Caixa, 2001. Colección estudios sociales, $n^{\circ} 6$.

3. Gènova R y Pereira J. Estudio monográfico: las expectativas de salud. En Las Personas mayores en España. Informe 2002. IMSERSO. Disponible en: http:/www.persomayores.csic.es/estadisticas/in formacion/ informe2002/index.html

4. Almenara Barios J, García Ortega C. Cambios demogáficos y asistencia geriátrica. En L Salvador Carulla, A. Cano Sánchez y JR Cabo Soler. Longevidad. Editorial Panamericana. En prensa.

5. Ribera Casado JM. Envejecimiento. En: Coca A. Envejecimiento, hipertensión y deterioro cognitivo. Libro digital. Disponible en: www.azprensa.com/biblioteca.php

6. Manton KG, Stallard E, Corder LS. The dynamics of dimensions of age-related disability 1982 to 1994 in the U.S. elderly population. J Gerontol A Biol Sci Med Sci 1998;53 (1): B59-70.

7. Manton $\mathrm{KG}, \mathrm{Gu} \mathrm{X}$. Changes in the prevalence of chronic disability in the United States black and nonblack population above age 65 from 1982 to 1999. Proc Natl Acad Sci U S A 2001; 98: 6354-6359.

8. Cruz Jentoft AJ. Cambios demográficos y asistencia geriátrica. Libro de ponencias del Aula de Debate Sociosanitaria 1998. Madrid: Aula Médica, 1998. p. 83-88.

9. Servicio Andaluz de Salud. Conjunto Mínimo Básico de Datos Andaluz (CMBDH-A). Sevilla: Consejería de Salud - Servicio Andaluz de Salud, 1994.

10. García Ortega C, Mérida de la Torre FJ, Almenara Barrios J, Zafra Mezcua J. Morbilidad hospitalaria aguda de las personas Mayores. Cádiz: Universidad de Cádiz, 2000.

11. Varela J, Castells X, Riu M, Cervera AM, Vernhes T, Diez A, et al. El impacto del envejecimiento sobre la casuística del hospital. Gac Sanit 2000; 14: 203-209.

12. Castells X, Mercadé LLuc y Riu M. Envejecimiento y utilización hospitalaria. En: Informe SESPAS 2002. Valencia, Consellería de Sanitat, 2002; 495-510.

13. Cruz Jentoft AJ. Características específicas del enfermo mayor. An Med Interna (Madrid) 2000; 17: 3-8.

14. Krumholz HM. Cardiopatía isquémica en el anciano. Conferencia Especial del XXXVI Congreso Nacional de la Sociedad Española de Cardio- logía. Rev Esp Cardiol 2001; 54: 819-826.

15. Guillén Llera, F. Hipertensión sistólica, riesgo vascular y edad avanzada. Geriatría y Gerontología 2001; 36: 65-68.

16. Ferreira Montero IJ. Insuficiencia cardiaca: mayor morbilidad, menor mortalidad.;Vamos por el buen camino? Rev Esp Cardiol 2000; 53: 767-769.

17. Matias-Guiu J. La epidemiología cerebrovascular. Rev Esp Cardiol 2000; 53: 13-14.

18. Tomás AbadalL, Puig T, Balaguer Vintró I. Accidente vascular cerebral: incidencia, mortalidad y factores de riesgo en 28 años de seguimiento. Estudio Manresa. Rev Esp Cardiol 2000; 53: 15-20.

19. Bayas JM, Vilella A, San-Martín M, González A, Conesa A, Asenjo MA. Impacto sanitario de los ingresos por neumonía en un hospital de tercer nivel. Med Clin (Barc) 2001; 116: 694 - 695.

20. Bonilla Rodríguez AB, Gómez Rodríguez MJ, Robles Agudo F, et al. Neumonía en el anciano institucionalizado: criterios de derivación y/o clasificación pronóstica. An Med Interna (Madrid) 2003; 20: 8-12.

21. Salleras L, Urbiztondo L,Fernández N, Comín E, Sánchez F, Batalla J et al. Vacunación antineumocócica en las personas mayores. Med Clin (Barc) 2001; 116 (Supl. 1): 18-23.

22. Fernández Torres B, García Ortega C, Márquez Espinós C, Fontán Atalaya M. Caracterización de la cirugía mayor ambulatoria en un hospital general básico. Rev Esp Salud Pública 1999; 73: 71-80.

23. Gili Miner M, Murillo Cabezas M, Perea-Milla López E. Epidemiología y prevención de los traumatismos. En: Martínez Navarro F, Antó JM, Castellanos PL, Gili M, Marset P, Navarro V. Salud Pública. Madrid: McGraw-Hill-Interamericana, 1997. p. 613-631.

24. Cabasés Hita JM, Carmona López G, Hernández Vecino R. Incidencia, riesgo y evolución de las fracturas osteoporóticas de cuello de fémur en las mujeres en España, a partir de un modelo de Markov. Med Clin (Barc) 2000; 114 (Supl. 2): 63-67.

25. Albert V, Maestro ME, Martínez JA, Monge V. Factores relacionados con la accidentabilidad en personas mayores de 65 años en la provincia de Guadalajara. Gac Sanit 2000; 14: 346-347.

26. Arboleya LR, Morales A, Fiter J. Efecto del alendronato sobre la densidad mineral ósea y la incidencia de fracturas en mujeres posmenopáusicas con osteoporosis. Un metanálisis de los estudios publicados. Med Clin (Barc) 2000; 114 (Supl. 2): 79-84.

27. Organización Panamericana de la Salud. Salud de las personas de edad. Envejecimiento y salud: un cambio de paradigma. Washington DC, 1998. CSP25/12. 